\title{
A neural network approach to dynamic job shop scheduling
}

\author{
T. Eguchi, F. Oba, T. Hirai \\ Faculty of Engineering, Hiroshima University \\ 1-4-1 Kagamiyama Higashi-Hiroshima City, 739-8527 \\ Japan \\ Tel\&Fax +81-824-24-7552 \\ e-mail: eguchi@mec.hiroshima-u.ac.jp
}

\begin{abstract}
In this study we propose a neural network approach to dynamic job shop scheduling problems. The aim of our approach is not to obtain the optimal schedule but to generate a good schedule in real-time as far as possible. Near-optimal schedules of small-scale problems are used to train the neural network. After training, the neural network is used to calculate each job's priority in real-time. Simulation experiments show the effectiveness of this approach to larger scale problems.
\end{abstract}

\section{Keywords}

Job shop, Dynamic scheduling, Real-time, Neural network, Due date, Weighted tardiness cost

\section{INTRODUCTION}

Scheduling is one of the key issues of the manufacturing systems. In particular, efficient scheduling for manufacturing systems such as job shops that produce a variety of products is crucially important in today's competitive markets. Although a vast amount of literature has been published within the last 30 years, it is difficult to generate even near-optimal schedules of practical scale because most job scheduling problems are proven to be NP-hard. Even if optimal schedules are obtained, the dynamic and stochastic nature of the real life situation makes preschedules brittle. Therefore researchers and practitioners have used dispatching rules to dynamic scheduling problem. Dispatching rules are easy to implement and computationally efficient. However, they cannot always guarantee desirable performance because the performance of dispatching rules is highly system 
dependent. Furthermore, the performance of simple dispatching rules is known to deteriorate as the scale of the problem increases.

In order to overcome these difficulties, various applications of neural networks have been proposed (Sabunchoglu, 1998). In these researches, the multi-layer feedforward neural networks are used to store human scheduler's expertise (Kitaoka et al., 1991) or the performance of appropriate dispatching rules in various situations (Sim et al., 1994). It is difficult to outperform the performance of human scheduler or best dispatching rules by this approach in the job shop environment.

This paper proposes a real-time scheduling method in a dynamic job shop environment by using a neural network. In our approach, a multi-layer feedforward neural network is used to calculate the priority of each job waiting in the input buffer of machine. The aim of this approach is not to obtain the optimal schedule (Jain and Meeran, 1998) but to generate a good schedule in real-time as far as possible. The training is carried out using small scale scheduling examples generated by a genetic algorithm. After training, the neural network is applied to larger scale problems. Simulation results show that the effectiveness of the proposed method to larger scale scheduling problems.

\section{NEURAL NETWORK APPROACH}

The performance measures of scheduling in this paper is the weighted tardiness cost per job, and is defined as

$$
\sum_{i=1}^{N} v_{i} \times \max \left(0, C_{i}-d_{i}\right) / N
$$

where $C_{i}, d_{i}, v_{i}$ are the completion time, due date, and delay penalty per unit time of job $_{i}$ respectively, and $N$ is the total number of jobs under consideration.

We focus on the next job selection to be processed from the jobs waiting service in the input buffer of machine. The neural network is applied to each waiting job to calculate the priority, and the job with the highest priority is selected as the next job to be processed. A three-layer feed-forward neural network is designed for this priority decision system.

When designing the neural network, there are three important points to achieve good performance: selection of the input information, training data generation, and training method of the neural network. These are described in the following sections.

\subsection{Input information to the Neural Network}

The input information to the neural network is very important in designing the network because it directly affects the decision of the priority. The appropriate selection of input information depends on the objective of scheduling and the shop floor characteristics to be applied. In this paper, we adopt four kinds of parameters as follows. 
1) Job information in the entire shop floor: Three input units are prepared to input this information. These are as follows: number of jobs in the current shop floor; rate of tardy jobs in the current shop floor; rate of critical jobs in the current shop floor. The tardy job means the job that has negative slack value. The critical job means the job that has positive slack but the value is lower than a predetermined control parameter.

2) Job information in the input buffer in which a job is to be selected: there are six units to input this information. These are as follows: number of jobs in the buffer; total processing time of the waiting jobs in the buffer; number of the tardy jobs in the buffer; number of the critical jobs in the buffer; sum of the slack value of tardy jobs; sum of the critical value of critical jobs.

3) Job information in the input buffer to which a job is transferred next. These are given by the same parameters as mentioned in 2). The number of units to input this kind of information is six.

4) Each job information: These are as follows: processing time; number of remaining process; remaining processing time; slack value; remaining allowance value $=$ due date - current time; delay penalty of the job per unit time $=v_{i}$. Two input units are prepared for each of slack and allowance value. One is used for positive value and another is for negative one. Therefore the total number of the input units for this kind of information is eight.

5) The relative value of the information of each job given in 4): These are given by the value of each job information divided by the maximum value among the all jobs in the buffer. Six input units are prepared for the six kinds of information described above. Negative values of the slack and allowance value are not considered. We also add another two relative values. Those are the relative number of jobs and the relative total processing time of jobs in the buffer of next machine to be processed.

All elements of the input information are transformed to take on numbers between 0 and 1. For example, the number of jobs in the current shop floor is divided by a scaling parameter that represents the maximum capacity of the shop.

\subsection{Training Data Generation}

The training data of the neural network is collected from sub-optimal schedules with regard to the weighted tardiness cost. Although arrival time of each job is not known when the trained neural network is used, we assume that it is known at the training phase. In such static environment, a genetic algorithm is used to obtain the sub-optimal schedules.

In this study, we restrict the search of optimal schedules in the non-delay schedules. And we take into consideration of the limitation of input buffer capacities. Genetic algorithms can easily cope with these constraints, if the chromosome is properly encoded. In our coding method (Eguchi et al., 1998), each process of the jobs has a gene that represents the priority value of the process. Crossover operation is conducted based on uniform crossover. Reproduction is accomplished by an elitist strategy. The fitness of each individual is measured by 
the weighted tardiness cost obtained by scheduling simulation using the priorities. Although this method is time consuming, the feasibility of all individuals is guaranteed if all constraints, such as buffer capacity limitations, are included in the scheduling simulator used to sequence the jobs. Another advantage of this coding method is that it is possible to obtain not only the sub-optimal schedules but also the priority of each job.

\subsection{Training of the Neural Network}

Training of the neural network is carried out by using the input-target pairs collected from the each situation of job selection in the sub-optimal schedules. The input is the shop floor status when a job is selected and target is the priority value of each job waiting for the service in the buffer. The priority value is directly obtained from a gene in the fittest individual obtained by the genetic algorithm.

Connection weights of the neural network are tuned based on the backpropagation algorithm. However, it is well known that the algorithm often falls into a local minimum of error function. It is crucially important to avoid getting stuck in poor local minima when training neural networks, especially when the solution space to be solved is very complicated. Various methods have been developed such as the hybrid algorithm that combines the modified back-propagation method and the random optimization method (Baba et al., 1994). In this paper, we adopt a simple hybrid algorithm as follows.

Step1. Set initial weights vector $\boldsymbol{w}^{(0)}$ using uniform distribution $[-W, W]$ Set parameter values of $M$ and $K$. Let $k=0$.

Step2. Update weights vector using back-propagation method.

This is carried out by the batch learning method of $M$ times.

Step3. Generate random noise vector $\xi u s i n g$ uniform distribution $[-W, W]$ Update weights data $\boldsymbol{w}^{(k)}$ as follows.

$$
\begin{aligned}
& \text { If } S\left(\boldsymbol{w}^{(k)}+\xi\right)<S\left(\boldsymbol{w}^{(k)}\right) \text { then } \boldsymbol{w}^{(k+1)}=\boldsymbol{w}^{(k)}+\xi \\
& \text { else if } S\left(\boldsymbol{w}^{(k)}-\xi\right)<S\left(\boldsymbol{w}^{(k)}\right) \text { then } \boldsymbol{w}^{(k+1)}=\boldsymbol{w}^{(k)}-\xi \\
& \text { else } \boldsymbol{w}^{(k+1)}=\boldsymbol{w}^{(k)}
\end{aligned}
$$

Step4. If $k=K$ then stop. Otherwise, let $k=k+1$ and go to step2.

$S(w)$ is the weighted tardiness cost of the schedule using the neural network with weight vector $\boldsymbol{w}$. For computing $S(\boldsymbol{w})$, we use the scheduling problems different from scheduling examples used to generate the training data, and the weights data that can achieve the best scheduling performance is selected as the final weights data.

\section{SIMULATION EXPERIMET}

\subsection{Scheduling Example for Training}

Numerical examples are presented to show the effectiveness of the proposed method. First, we generate scheduling examples for training the neural network. In 
the examples, each machine in the shop has an input buffer that can store up to 3 jobs. If an input buffer is full, the jobs that are going to the buffer are transferred to a central buffer and wait until the buffer becomes available. The assignment of operation to the machines is determined randomly. Other parameters are as follows.

- Number of machines: 4

- Number of jobs: 100

- Number of operations per job: discrete uniform distribution $[2,4]$

- Processing time for each operation: discrete uniform distribution $[5,100]$

- Due date $=$ arrival time of the job $+0.1 \times k_{\mathrm{d}} \times$ total processing time of the job

- $k_{\mathrm{d}}$ is determined by discrete uniform distribution $[30,40]$

- Delay penalty of job per unit time: discrete uniform distribution [1,5]

- 10 jobs are in the shop initially and the other jobs arrive randomly according to a Poisson process with arrival rate $\lambda=1 / 50$

Ten scheduling problems are generated and solved using the genetic algorithm to obtain the scheduling examples for training.

\subsection{Training of the Neural Network}

The neural network used in this paper has 31 input units, 10 hidden units, and one output unit. From the 10 scheduling examples, we collected 4273 input-target pairs from the each situation of job selection and used them for training. $W$ is set to 0.1 and the number of back-propagation $M$ is set to 100 . The learning rate of the backpropagation is set to 0.01 . Ten scheduling examples, each of which consists of 4 machines and 1000 jobs, are used to test the performance of the neural network during the training. Figure 1 shows the learning curve. The learning curves using the back-propagation and the random optimization method separately are also depicted. This figure shows that using the back-propagation method alone gives a very poor training result. Although random optimization method plays the main role to search good weights data, the hybrid method can find the better weights data. The weights data when $k=652$ is finally adopted.

\subsection{Experimental Results}

Larger scale scheduling problems were newly generated to evaluate the performance of the trained network. The scheduling parameters different from those used in the training phase are as follows. 


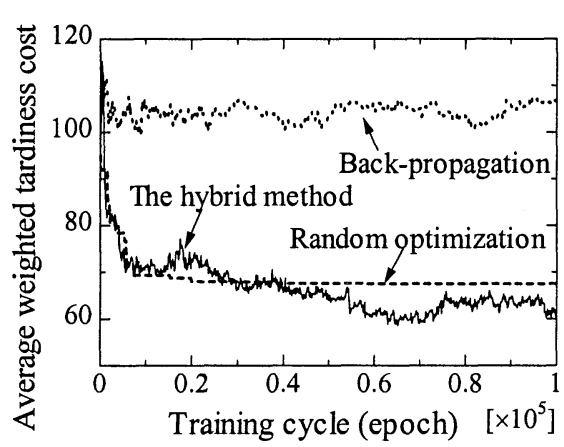

Figure 1 Learning curve

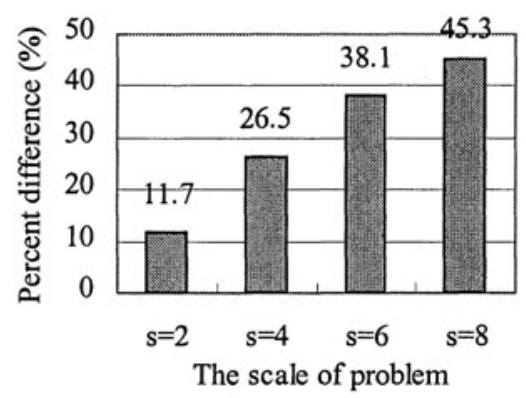

Figure 2 Percent difference between the performances of the proposed method and the weighted $\mathrm{CR}+\mathrm{SPT}$ rule

- Number of machines: $4 \times s$

- Number of jobs: 1000

- Number of operations per job: discrete uniform distribution $\left[2 \times_{S}, 4 \times S\right]$

- The shop is initially empty, and each job arrives randomly according to

a Poisson process with arrival rate $\lambda=1 / 50$

Four scales of problems $(s=2,4,6,8)$ are generated. For each scale problem, 100 scheduling problems are generated and used to evaluate the performance. For the evaluation, we select 500 jobs from the 1000 jobs in each scheduling problem. The first 1-250 jobs arrived earlier and the last finished 751-1000 jobs are discarded to remove the edge effects during the start-up and the termination stage of the simulation run. When the 500 jobs are going through the shop, the average machine utilization is $79 \square 80 \%$ for all scale problems.

The scheduling results by the proposed method are compared with those of single dispatching approach. Four dispatching rules, WSPT, ATC (Vepsalainen and Morton, 1987), Weighted CR+SPT and Weighted S/RPT+SPT (Anderson and Nyirenda, 1990) are tested because they are reported effective in the literature (Kutanoglu and Sabuncuoglu, 1999). The ATC rule has two parameters. Various values are tested for the parameters and the parameters recommended in the literature $(b=2, k=3)$ were found optimal for our scheduling problems. The other dispatching rules have no parameters to be tuned.

We have to note that some of the input data are divided by $s$ to transform input information to take on numbers between 0 and 1 . For example, when training using small-scale problems, the number of jobs in the current shop floor is divided by 20 before inputting to the neural network. For the larger problem with $s$, it is divided by $20 \times s$.

The simulation results are shown in Table 1 . The results show that the smallest weighted tardiness cost per job was given by the proposed method (N.N.) with regard to the average value of 100 scheduling results. The Weighted CR+SPT rule 
Table 1 Experimental Results (Average weighted tardiness cost per job)

\begin{tabular}{llccl}
\hline \multirow{2}{*}{ Scheduling rule } & $s=2$ & \multicolumn{4}{c}{ The scale of problem } & \multicolumn{1}{c}{$s=6$} \\
\hline WSPT & 172.9 & 260.7 & 327.3 & 364.5 \\
ATC & 105.9 & 112.9 & 92.5 & 61.6 \\
Weighted S/RPT+SPT & 106.2 & 122.9 & 106.7 & 89.4 \\
Weighted CR+SPT & 104.0 & 114.7 & 100.1 & 86.0 \\
N.N. & 91.8 & 84.3 & 62.0 & 47.0 \\
\hline
\end{tabular}

performed best among the single dispatching rules when $s=2$. The weighted tardiness cost generated by N.N. is $11.7 \%$ less than that generated by the Weighted $\mathrm{CR}+\mathrm{SPT}$ rule. When $s=8$, it is $45.3 \%$. Although the Weighted CR+SPT rule has an advantage of having no tuning parameter, the performance difference between the rule and the proposed method increases as the scale of problem increases (see Fig.2). When the scale of problem is large, such as $\mathrm{s}=6$ and 8 , the Weighted $\mathrm{CR}+\mathrm{SPT}$ rule can not outperform the ATC rule. These results may mean that to utilize parameters which represent the current status of jobs or shop floor has more effects when the scale of problem is large.

For all the problems, the difference between the proposed method and single dispatching rules was significant at a level $\alpha=0.01$ by the paired- $t$ confidence interval method (Law and Kelton, 1991). Furthermore, for example, when $s=4$, the proposed method outperformed the single dispatching rules in the 83 schedules among the 100 ones. In the rest 17 schedules, the weighted tardiness cost of N.N. was only 9.2 point larger than the best one given by single dispatching rules in the mean value. These results suggest that the proposed method has a great effect on the performance of larger scale job shop scheduling.

\section{CONCLUSION}

A dynamic job shop scheduling method by using a neural network was proposed in this paper. The simulation results showed the effectiveness of the proposed method to the larger scale problems. Further research is necessary to evaluate the robustness for the various situations that are different from ones used to train the neural network.

\section{REFERENCES}

Anderson,E.J. and Nyirenda,J.C., (1990) Two New Rules to Minimize Tardiness in a Job Shop, International Journal of Production Research, 28, 2277-2292 
Baba,N., Mogami,Y., Kohzaki,M., Shiraishi,Y., and Yoshida,Y.,(1994) A Hybrid Algorithm for Finding the Global Minimum of Error function of Neural Networks and Its Application, Neural Networks,7,1253-1265

Eguchi,T., Oba,F. and Hirai,T. (1998) Dynamic Job Shop Scheduling with a Neural Network and a Genetic Algorithm, Proceedings of 1998 Japan-U.S.A Symposium on Flexible Automation, $\square, 1393 \square 1396$

Jain,A.S. and Meeran,S. (1998) Job-shop scheduling using neural network, Journal of Production Research, 36,1249-1272.

Kitaoka,M., Dong,Y. and Tsuchida,K.,(1991) Design of the Production Scheduling System for a Corrugated Cardboard Factory Using Neural Networks, Management System, 1, 72-80. (in Japanese)

Law,A.M., and Kelton,W.D., (1991) in Simulation Modeling $\square$ analysis, 2nd Edition, McGraw-Hill,Inc.

Sabunchoglu,I.,(1998) Scheduling with Neural Networks : A Review of the Literature and New Research Directions, Production Planning $\square$ Control, 9, 212.

Sim,S.K.,Yeo,K.Y. and Lee,W.H.,(1994) An Expert Neural Network System for Dynamic Job Shop Scheduling, International Journal of Production Research , 32, 1759-1773.

Vepsalainen,A.P.J. and Morton,T.E., (1987) Priority Rules for Job Shops with Weighted Tardiness Costs, Management Science, 33, 1035-1047.

\section{BIOGRAPHY}

Toru Eguchi is a research associate at Hiroshima University. He received the M.S. degree in engineering from Hiroshima University, Japan, in 1993. His current research interests include planning and scheduling of manufacturing systems.

Fuminori Oba is a professor at Hiroshima University since 1988. He received his master degree from University of Osaka Prefecture in 1968 and his doctor degree from Osaka University in 1975. His current research interests include design of manufacturing systems, scheduling, and production management.

Toshiki Hirai received the M.S. degree in engineering from Hiroshima University, Japan, in 1999. He currently works for Sekisui Systems Corporation. 